\title{
Using an obliquely incident laser beam to measure optical properties of turbid media
}

Lihong V. Wang, Steven L. Jacques

Lihong V. Wang, Steven L. Jacques, "Using an obliquely incident laser beam to measure optical properties of turbid media," Proc. SPIE 2389, Optical Tomography, Photon Migration, and Spectroscopy of Tissue and Model Media: Theory, Human Studies, and Instrumentation, (30 May 1995); doi: $10.1117 / 12.210001$

SPIE. Event: Photonics West '95, 1995, San Jose, CA, United States 


\title{
Using an Obliquely Incident Laser Beam to Measure Optical Properties of Turbid Media
}

\author{
Lihong Wang, Steven L. Jacques \\ Laser Biology Research Laboratory, Box 17 \\ University of Texas M. D. Anderson Cancer Center \\ 1515 Holcombe Boulevard \\ Houston, Texas, USA
}

\begin{abstract}
A simple and quick approach was invented to measure optical properties of tissue-like turbid media. A laser beam with oblique incidence to the medium causes the center of the diffuse reflectance that is several transport mean free paths away from the incident point to shift from the point of incidence. The amount of shift is used to compute the reduced scattering coefficient by a simple formula. This formula is a function of the refractive index of the turbid medium divided by that of the incident medium and the angle of incidence off the surface normal for a semi-infinite turbid medium having a much smaller absorption coefficient than the reduced scattering coefficient. For a turbid medium having a comparable absorption coefficient with the reduced scattering coefficient, a revision to the above formula was made. The slope of the diffuse reflectance can be used to compute the penetration depth. Both the computation of the reduced scattering coefficient and penetration depth are based on simple and quick algorithms. The validity conditions of the algorithms for slabs of turbid media are studied. This technique has potential for non-invasive, in vivo, real-time diagnosis of disease or monitoring of treatments.
\end{abstract}

Key words: Tissue optics, turbid media, scattering media, absorption coefficient, reduced scattering coefficient, optical properties, Monte Carlo simulation. 


\section{GLOSSARY}

\begin{tabular}{|c|c|}
\hline $\begin{array}{l}\text { Absorption coefficient } \\
{\left[\mu_{\mathrm{a}}, \mathrm{cm}^{-1}\right]}\end{array}$ & The probability of photon absorption per unit infinitesimal pathlength. \\
\hline Anisotropy $[\mathrm{g}]$ & $\begin{array}{l}\text { The average of the cosine value of the deflection angle by single } \\
\text { scattering. }\end{array}$ \\
\hline $\begin{array}{l}\text { Effective attenuation } \\
\text { coefficient }\left[\mu_{\mathrm{eff}}, \mathrm{cm}^{-1}\right]\end{array}$ & $\begin{array}{l}\text { The decay constant of light fluence far away from light source. } \mu_{\mathrm{eff}}= \\
\sqrt{3 \mu_{\mathrm{a}} \mu_{\mathrm{t}}{ }^{\prime}}=\sqrt{3 \mu_{\mathrm{a}} \mu_{\mathrm{s}}(1-\mathrm{g})} \text {. }\end{array}$ \\
\hline $\begin{array}{l}\text { Interaction coefficient } \\
{\left[\mu_{\mathrm{t}}, \mathrm{cm}^{-1}\right]}\end{array}$ & $\begin{array}{l}\text { The probability of photon interaction per unit infinitesimal pathlength, } \\
\text { where the interaction includes both absorption and scattering. } \mu_{\mathrm{t}}=\mu_{\mathrm{a}} \\
+\mu_{\mathrm{s}} \text {. Sometimes, it is also called total interaction coefficient or total } \\
\text { attenuation coefficient. }\end{array}$ \\
\hline Mean free path $[\mathrm{mfp}]$ & The mean pathlength between interactions, which is $1 / \mu_{\mathrm{t}}$. \\
\hline $\begin{array}{l}\text { Penetration depth }[\delta \text {, } \\
\mathrm{cm}]\end{array}$ & $1 / \mu_{\text {eff. }}$ It represents decay constant of the light fluence in the far-field. \\
\hline $\begin{array}{l}\text { Scattering coefficient } \\
{\left[\mu_{\mathrm{s}}, \mathrm{cm}^{-1}\right]}\end{array}$ & The probability of photon scattering per unit infinitesimal pathlength. \\
\hline $\begin{array}{l}\text { Transport interaction } \\
\text { coefficient }\left[\mu_{\mathrm{t}}^{\prime}, \mathrm{cm}^{-1}\right]\end{array}$ & $\mu_{\mathrm{t}}^{\prime}=\mu_{\mathrm{a}}+\mu_{\mathrm{s}}{ }^{\prime}$ \\
\hline $\begin{array}{l}\text { Transport mean free } \\
\text { path [mfp'] }\end{array}$ & $1 / \mu_{\mathrm{t}}^{\prime}$ \\
\hline $\begin{array}{l}\text { Transport scattering } \\
\text { coefficient }\left[\mu_{\mathrm{s}}^{\prime}, \mathrm{cm}^{-1}\right]\end{array}$ & $\begin{array}{l}\mu_{\mathrm{s}}{ }^{\prime}=\mu_{\mathrm{s}}(1-\mathrm{g}) \text {. Sometimes, it is also called reduced scattering } \\
\text { coefficient. }\end{array}$ \\
\hline
\end{tabular}

\section{INTRODUCTION}

In both diagnostic and therapeutic applications of lasers or other light sources in medicine, it is important to evaluate optical properties of biological tissues. The optical properties can be 
used to diagnose diseases, to measure tissue metabolic status, or to determine the dosimetry in therapeutic applications of lasers. Non-invasive, in vivo, real-time measurements of tissue optical properties $\left(\mu_{\mathrm{a}}\right.$ and $\left.\mu_{\mathrm{s}}^{\prime}\right)$ remain a challenge.

A typical diffuse reflectance of a laser beam normally incident upon a semi-infinite turbid medium as a function of the distance between the observation point and the incident point of the laser beam, i.e., $\mathrm{R}(\mathrm{x})$, is shown in Fig. 1. Monte Carlo simulated diffuse reflectance is considered accurate, whereas the diffusion theory predicted diffuse reflectance is only accurate when the observation point is several transport mean free paths away from the incident point of the laser beam. For simplicity, we will define the diffuse reflectance that is several mfp' away from the incident point of the laser beam as far diffuse reflectance. Conversely, we will define the diffuse reflectance that is within several $\mathrm{mfp}^{\prime}$ away from the incident point of the laser beam as near diffuse reflectance.

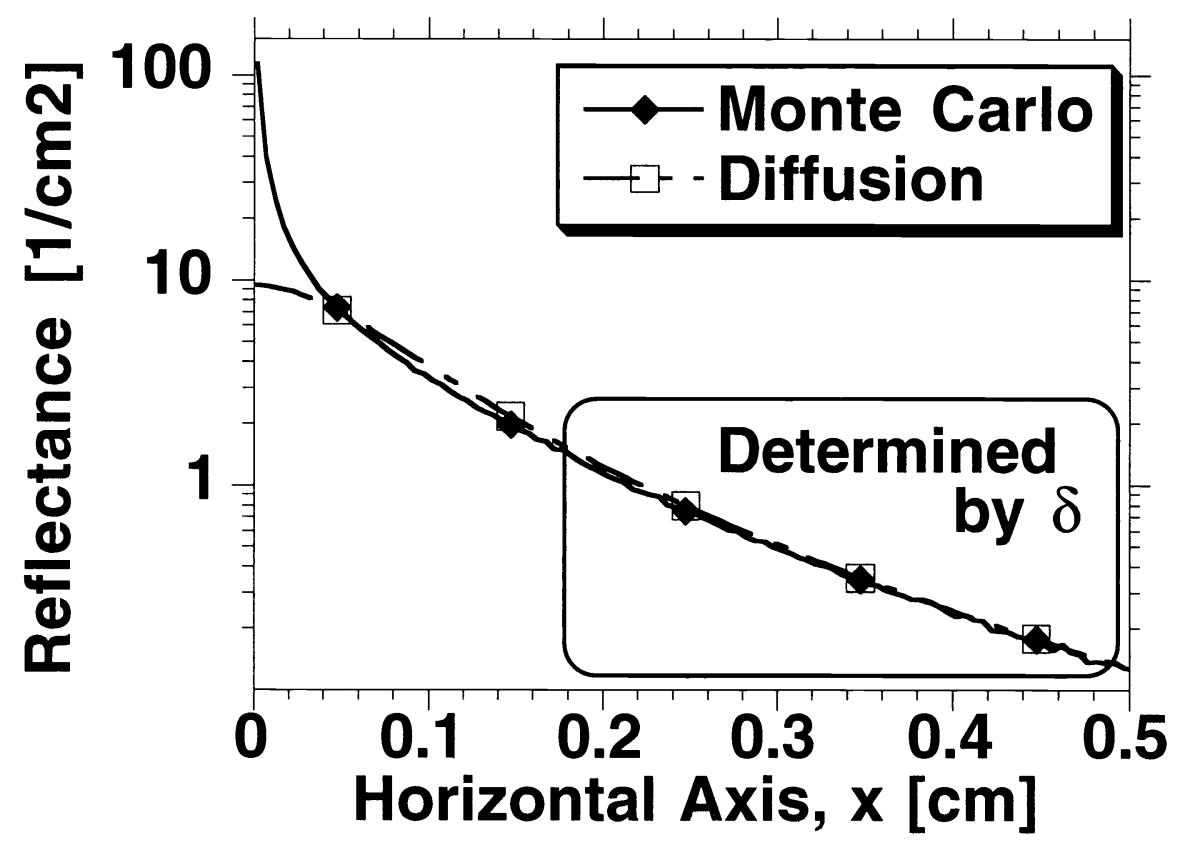

Fig. 1. Diffuse reflectance of a laser beam normally incident upon a semi-infinite turbid medium as a function of $\mathrm{x}$, which is the distance between the observation point and the incident point of the laser beam (see Fig. 2). The computation was based on Monte Carlo simulation and diffusion theory. The optical properties of the turbid medium are index of refraction $\mathrm{n}=1.0$, absorption coefficient $\mu_{\mathrm{a}}=0.1 \mathrm{~cm}^{-1}$, scattering coefficient $\mu_{\mathrm{s}}=100$ $\mathrm{cm}^{-1}$, anisotropy $\mathrm{g}=0.9$. 
The far diffuse reflectance becomes linear on the semi-logarithmic plot, and the slope of the diffuse reflectance is primarily determined by the penetration depth $\delta$. If one measures the relative profile of the diffuse reflectance $\mathrm{R}(\mathrm{x})$, the penetration depth can be computed using a relatively simple algorithm based on the slope of the reflectance. ${ }^{1}$ If we wish to measure both absorption and scattering coefficients, two independent parameters have to be determined. The near diffuse reflectance of the relative profile has to be used to determine another independent parameter, which creates a dilemma. No simple algorithm is available to deduce the second parameter from the near diffuse reflectance. Algorithms based on Monte Carlo simulations will be time-consuming because of the stringent demand of computation time of Monte Carlo simulations. Neural network may be used to solve the inverse problem after the network is trained on a set of Monte Carlo simulation results of various optical properties. ${ }^{1,2}$ Once the neural network is trained, the inverse problem can be solved in real-time. However, the neural network approach involves a long training process.

Algorithms based on diffusion theory cannot use the near diffuse reflectance because of the inaccuracy of diffusion theory in the near region. If the absolute far diffuse reflectance is measured, the diffusion theory based algorithms should be able to deduce the second parameter using non-linear least squares fits of the far diffuse reflectance. However, it is much easier to measure the relative profile of diffuse reflectance than to measure the absolute profile.

We have introduced a simple method that deduces $\mu_{\mathrm{s}}$ ' very quickly and is independent of the laser beam size if the laser beam has a predefined mirror symmetry and the size of the beam is smaller than the distance between the observation points and the center of the laser beam. ${ }^{3}$ For example, a circular flat beam or a circular Gaussian beam has an elliptic spot on the medium surface when the beam is incident at an oblique angle. The mirror symmetry of this elliptic spot about its short axis allows the application of this method to measure $\mu_{\mathrm{s}}$. The method was verified theoretically and experimentally.

Here we will present more modeling results based on Monte Carlo simulation results. The slope of the far diffuse reflectance of oblique incidence is compared with that of normal incidence. The validity conditions of the algorithms for slabs of turbid media are studied.

\section{METHODS}

Monte Carlo simulations have been used to compute the diffuse reflectance of a laser beam incident upon a turbid medium. ${ }^{4,5}$. Our Monte Carlo simulation package, which can run on any computer platforms supporting ANSI C (such as Sun, Mac, IBM PC/compatible), can be obtained from the authors (Email: lihong@laser.mda.uth.tmc.edu) or downloaded directly from our anonymous ftp site. The ftp address is laser.mda.uth.tmc.edu (129.106.60.92). 
A Cartesian coordinate system is placed on the turbid medium (Fig. 2). The origin of the coordinate system is at the incident point of the laser beam that is assumed infinitely narrow for the computation.

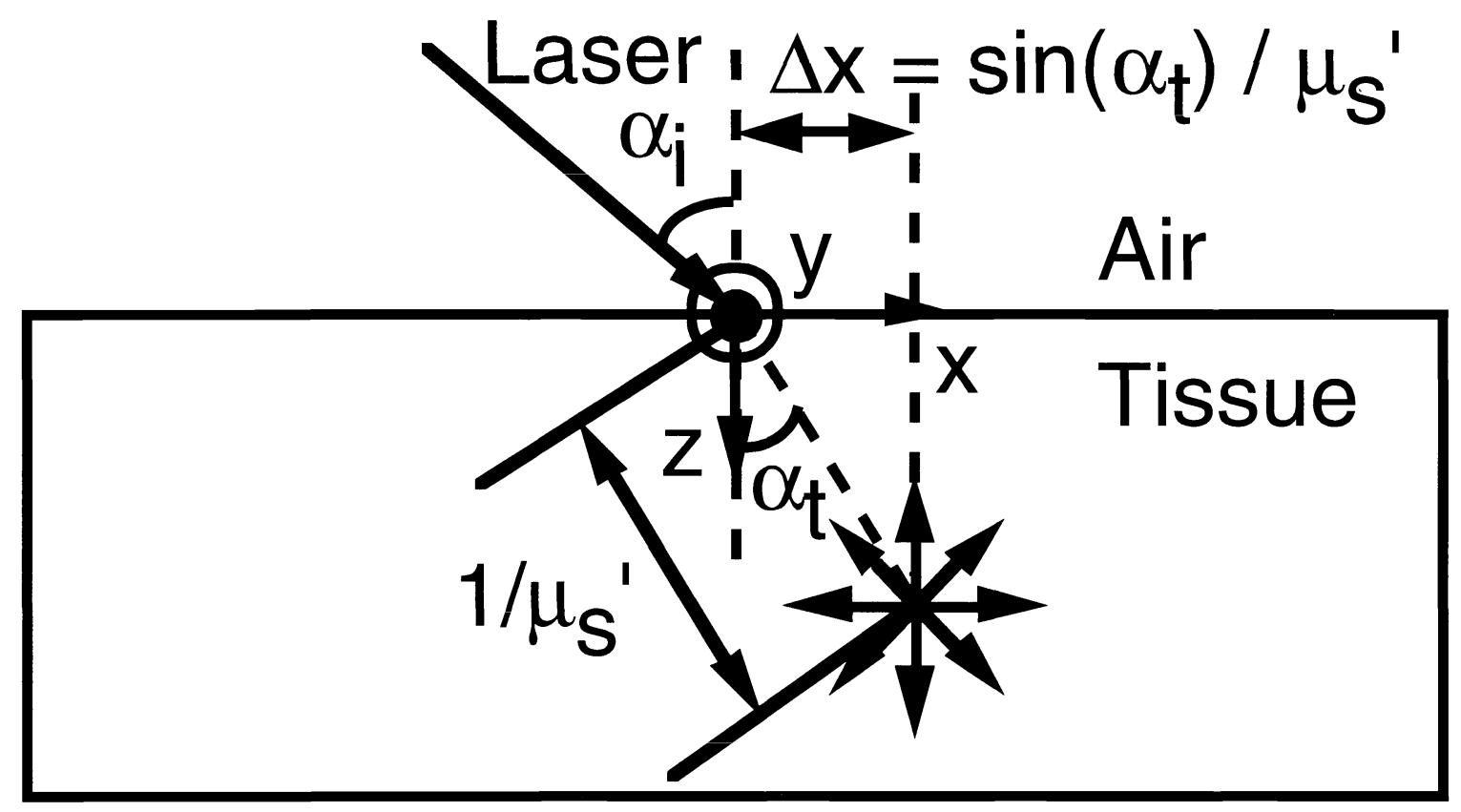

Fig. 2. Coordinate system and the lumped interaction approximation of the laser beam. In the figure, $\alpha_{i}$ is the incident angle of the laser beam, $\alpha_{t}$ is the refracted angle, $\Delta x$ is the horizontal shift of the lumped interaction site from the incident point. The y-axis point outward from the paper.

\section{RESULTS}

If a laser beam is normally incident upon a semi-infinite turbid medium, the diffuse reflectance will resemble the plot shown in Fig. 3. For symmetry reasons, the diffuse reflectance on the plot demonstrates concentric rings (contour lines) centered at the incident point of the laser beam.

If a laser beam is obliquely incident upon a semi-infinite turbid medium, the diffuse reflectance will resemble the plot shown in Fig. 4. Near the origin of the xy-plane (incident point of the laser beam), the diffuse reflectance is asymmetric. Far away from the origin, the diffuse reflectance becomes symmetric again, and there is an apparent center for the concentric rings (contour lines) in the far region. The apparent center is shifted toward the $+\mathrm{x}$ direction. 

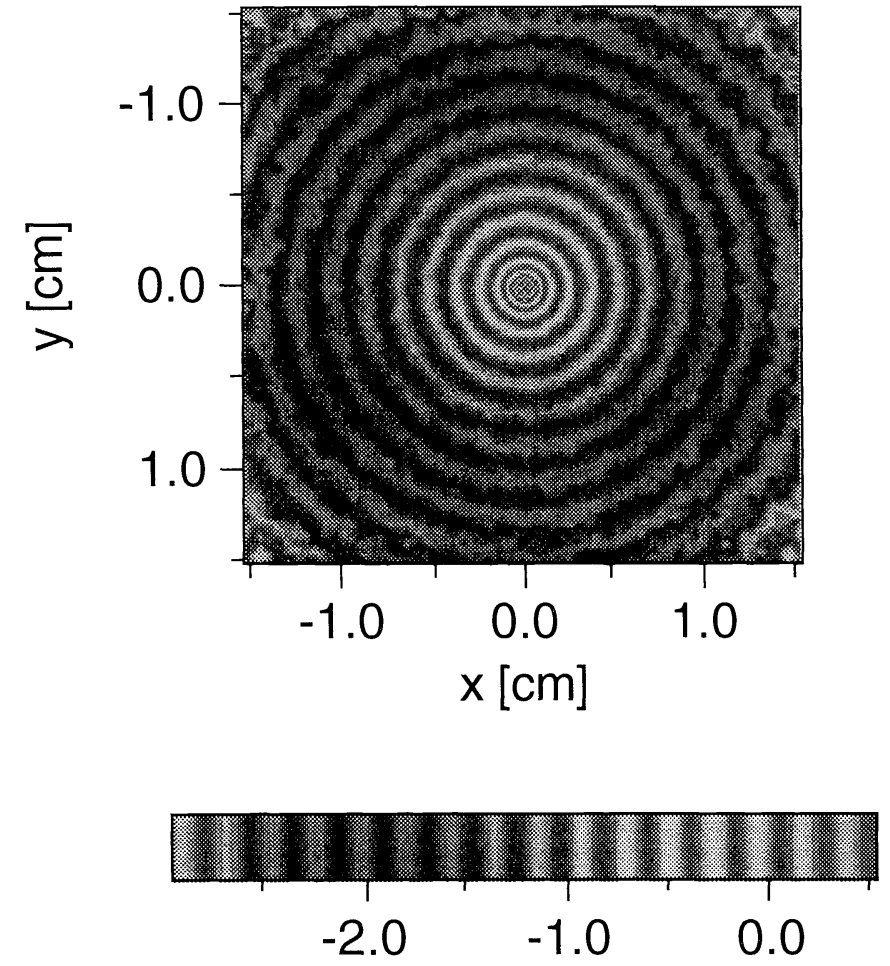

Log of Reflectance
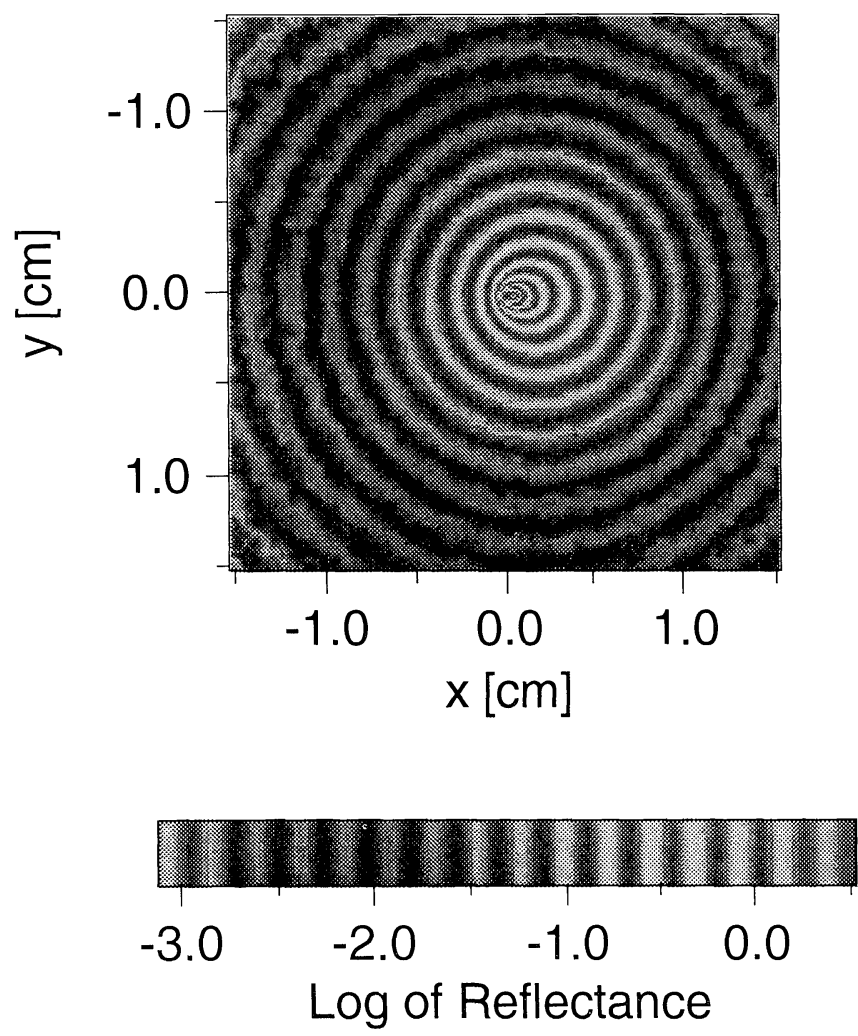

Fig. 3. 2D distribution of diffuse reflectance of a normally incident laser beam $\left(\alpha_{i}=0\right)$ computed with Monte Carlo simulations. Each ring represents a contour line of diffuse reflectance. The bottom bar is the palette used to generate the plot. The optical properties of the semiinfinite turbid medium are index of refraction $n=1.33$, absorption coefficient $\mu_{\mathrm{a}}=$ $0.25 \mathrm{~cm}^{-1}$, scattering coefficient $\mu_{\mathrm{s}}=20 \mathrm{~cm}^{-1}$, anisotropy $\mathrm{g}=0.853$.

Fig. 4. 2D distribution of diffuse reflectance of an obliquely inc: tent laser beam $\left(\alpha_{i}=45^{\circ}\right)$ computed with Monte Carlo simulations. Each ring represents a contour line of diffuse reflectance. The bottom bar is the palette used to generate the plot. The optical properties of the semiinfinite turbid medium are index of refraction $n=1.33$, absorption coefficient $\mu_{\mathrm{a}}=$ $0.25 \mathrm{~cm}^{-1}, \quad$ scattering coefficient $\mu_{\mathrm{s}}=20 \mathrm{~cm}^{-1}$, anisotropy $\mathrm{g}=0.853$. 
The diffuse reflectance distributions along the $\mathrm{x}$-axis in Figs. $3 \& 4$ are shown in Fig. 5 . The diffuse reflectance of normal incidence is symmetric about the incident point, whereas the diffuse reflectance of the oblique incidence is shifted toward the $+x$ direction. The slopes of the two far diffuse reflectance curves are parallel. When the diffuse reflectance of the oblique incidence is shifted, its far diffuse reflectance matches that of normal incidence. The amount of shift is computed by

$$
\Delta \mathrm{x}=\frac{\sin \left(\alpha_{\mathrm{i}}\right)}{\mathrm{n}\left(\mu_{\mathrm{s}}{ }^{\prime}+0.35 \mu_{\mathrm{a}}\right)},
$$

as derived in our previous publication. ${ }^{3}$. Eq. (1) is a refined version of the equation shown in Fig. 2 .

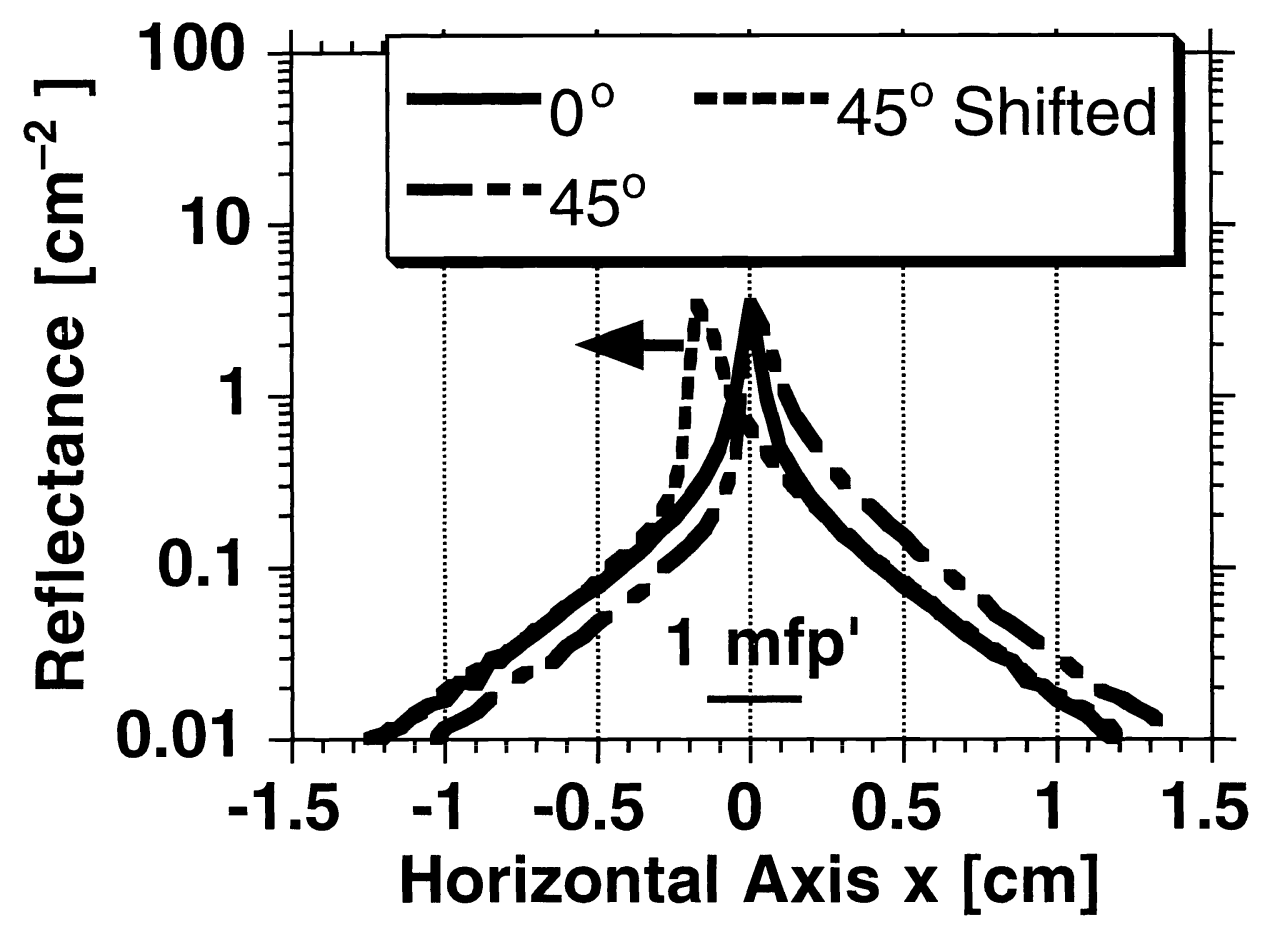

Fig. 5. The diffuse reflectance distributions along the x-axis in Figs. $3 \& 4$. The slopes of the two curves become parallel far away from the incident point of the laser beam. Once the diffuse reflectance curve of the 45 degree incidence was translated, its slope matches that of the diffuse reflectance curve of the normal incidence.

The validity of using Eq. (1) for tissue slabs of various thicknesses is tested (Fig. 6). When the index of refraction of the slab matches that of the ambient medium $(n=1.0)$, Eq. (1) can be used for slabs of thickness greater than $1 \mathrm{mfp}$. When the index of refraction of the slab 
mismatches that of the ambient medium $(n=1.37)$, Eq. (1) can be used for slabs of thickness as small as $1 / 10 \mathrm{mfp}$.

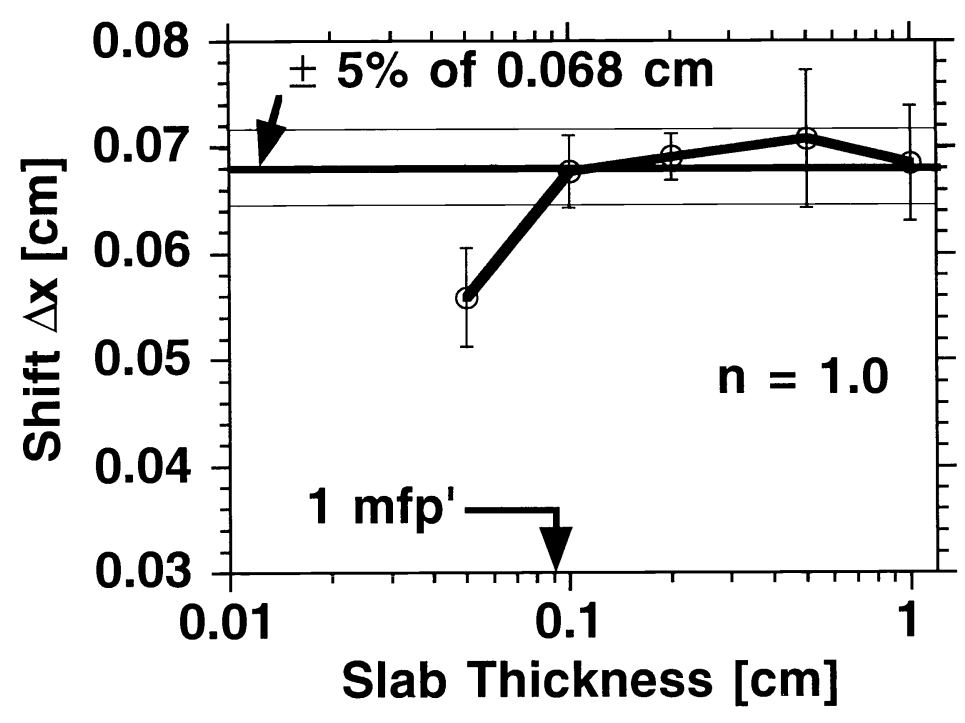

(a)

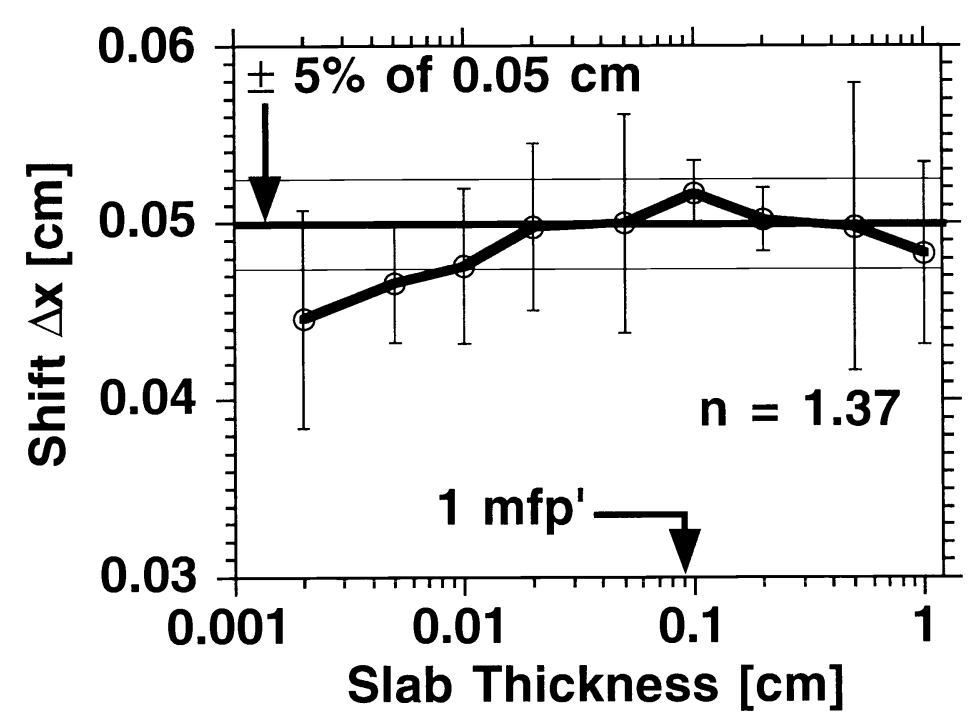

(b)

Fig. 6. The Monte Carlo simulated shift values $(\Delta \mathrm{x})$ of diffuse reflectance versus slab thicknesses, where the angle of incidence of the laser beam is $45^{\circ}$. The optical properties of the turbid medium are index of refraction (a) $n=1.0$ and (b) $n=1.37$, absorption coefficient $\mu_{\mathrm{a}}=1 \mathrm{~cm}^{-1}$, scattering coefficient $\mu_{\mathrm{s}}=100 \mathrm{~cm}^{-1}$, anisotropy $\mathrm{g}=0.9$. The shift values based on Eq. (1) are $0.068 \mathrm{~cm}$ and $0.05 \mathrm{~cm}$ for (a) and (b), respectively. 
The slope of diffuse reflectance of a slab will differ from that of semi-infinite turbid medium. The comparison between the diffuse reflectance of a laser beam normally incident upon a semi-infinite turbid medium and that of a laser beam obliquely incident upon a slab is shown in Fig. 7. For both refractive-index matched $(n=1.0)$ and mismatched $(n=1.37)$ turbid media, the far diffuse reflectance is parallel with that of a semi-infinite turbid medium when the slab thickness is greater than approximately $3 \delta$.

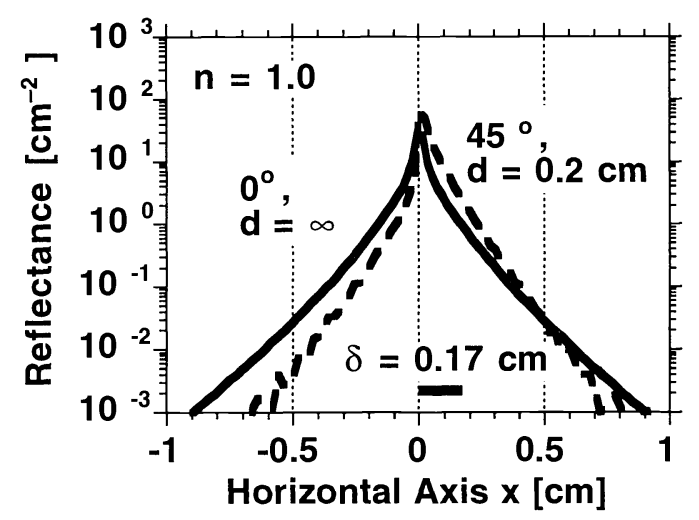

(a)

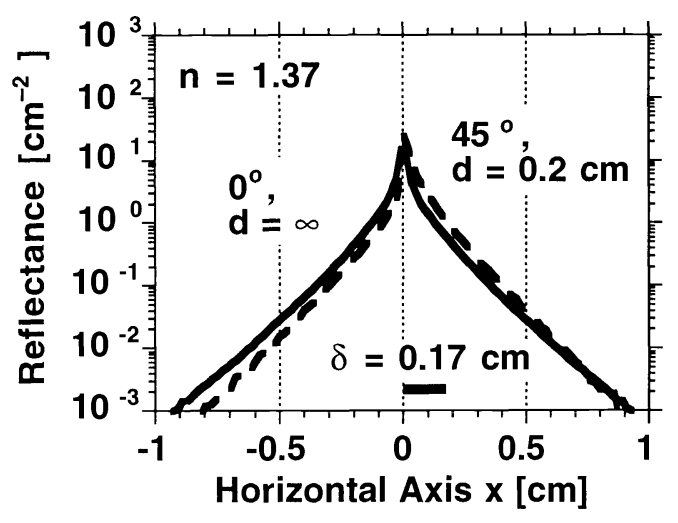

(c)

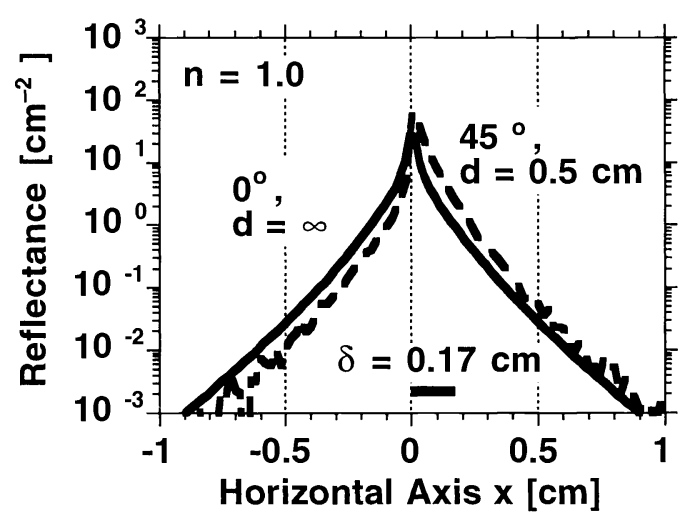

(b)

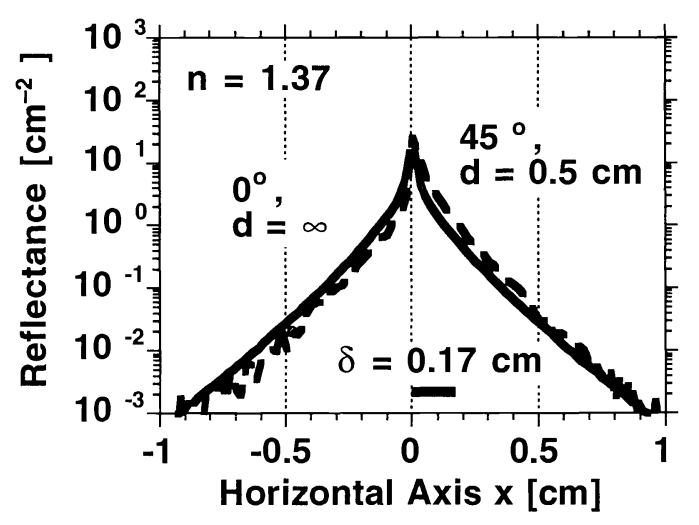

(d)

Fig. 7. Comparison between the diffuse reflectances of a laser beam normally incident upon a semi-infinite turbid medium and a laser beam obliquely incident upon a slab. The relative refractive index and the thickness of the turbid medium are shown in each figure. The optical properties of the turbid medium are absorption coefficient $\mu_{\mathrm{a}}=1 \mathrm{~cm}^{-1}$, scattering coefficient $\mu_{\mathrm{s}}=100 \mathrm{~cm}^{-1}$, anisotropy $\mathrm{g}=0.9$.

\section{CONCLUSION}

We have established in a previous publication ${ }^{3}$ that the shift of the apparent center of the far diffuse reflectance from the incident point of the laser beam yields the reduced scattering coefficient 
of the semi-infinite medium when the absorption coefficient is much less than the reduced scattering coefficient $\left(\mu_{\mathrm{a}}<<\mu_{\mathrm{s}}{ }^{\prime}\right)$, where the computation is based on the equation shown in Fig. 2. The equation was revised to Eq. (1) when $\mu_{\mathrm{a}}$ is comparable with $\mu_{\mathrm{s}}{ }^{\prime}$. In this case, Eq. (1) can be combined with another equation deduced from the slope of the far diffuse reflectance to determined both $\mu_{\mathrm{a}}$ and $\mu_{\mathrm{s}}{ }^{\prime}$.

This article has shown that the slope of the far diffuse reflectance of an obliquely incident laser beam is parallel with that of a normally incident laser beam upon a semi-infinite turbid medium (Figs. 3-5). Therefore, the simple technique that was used to deduce the penetration depth $\delta$ in the case of normal incidence can be used similarly in the case of oblique incidence. ${ }^{1}$

Therefore, the oblique incidence of a laser beam upon a semi-infinite medium allows determination of two independent optical parameters using one measurement of the diffuse reflectance. Both parameters can be computed from the relative profile of the diffuse reflectance, eliminating the need for absolute measurement of the diffuse reflectance.

When a slab of turbid medium is the target sample, the diffuse reflectance will be certainly different from that of a thick slab (semi-infinite turbid medium). The lumped interaction site in Fig. 2 will be moved up because the distribution of first interactions along the refracted laser beam is shortened by the bottom boundary. The slope of the diffuse reflectance will be affected by the bottom boundary as well.

The preliminary studies (Fig. 6) showed that when the index of refraction of the slab matches that of the ambient medium $(n=1.0)$, Eq. (1) can be used for slabs of thickness greater than $1 \mathrm{mfp}$. When the index of refraction of the slab mismatches that of the ambient medium $(\mathrm{n}=$ 1.37), Eq. (1) can be used for slabs of thickness as small as 1/10 $\mathrm{mfp}$. The internal reflection between the refractive index mismatched boundaries apparently reduced the minimal thickness of slabs required to accurately use Eq. (1).

Fig. 7 showed that for both refractive-index matched $(n=1.0)$ and mismatched $(n=1.37)$ turbid media, the far diffuse reflectance is parallel with that of a semi-infinite turbid medium when the slab thickness is greater $3 \delta$. Based on diffusion theory, the total amount of light that reaches $\mathrm{z}$ $=3 \delta$ is approximately $\mathrm{e}^{-3}=5 \%$ of the incident light. Therefore, the contribution of the light that has reached $\mathrm{z}=3 \delta$ to the diffuse reflectance may be neglected.

An optical fiber bundle, as opposed to the CCD camera system that was used in our original experiments, ${ }^{3}$ can be used to implement this technique. The optical fiber bundle approach should have the advantage of low-cost, ease of manipulation in clinical settings, and endoscopic applications. Both algorithms of deducing the optical parameters are simple and rapid. The optical fiber bundle approach may provide a real-time, non-invasive, in vivo tool for diagnosis of diseases, monitoring of physiological parameters such as hemoglobin oxygenation status, and monitoring of treatments of diseases. 


\section{ACKNOWLEDGMENT}

This research was supported in part by the Whitaker Foundation, Office of Naval Research grant N00014-91-J-1354, Air Force Office of Scientific Research grant F49620-93-1-0298DEF, Department of Energy grant DE-FG05-91ER61226, and National Institutes of Health grant R29HL45045.

\section{REFERENCES}

1. T. J. Farrell, B. C. Wilson, and M. S. Patterson, "The use of a neural network to determine tissue optical properties from spatially resolved diffuse reflectance measurements," Phys. Med. Biol. 37, 2281-2286 (1992).

2. L.-H. Wang, X.-M. Zhao, and S. L. Jacques, "Computation of the optical properties of tissues from light reflectance using a neural network," Proc. Soc. Photo-Opt. Instrum. Eng. 2134, 391-399 (1994).

3. L.-H. Wang and S. L. Jacques, "Use of a laser beam with an oblique angle of incidence to measure the reduced scattering coefficient of a turbid medium," Appl. Opt. 34, in press (1995).

4. L.-H. Wang and S. L. Jacques, Monte Carlo Modeling of Light Transport in Multi-layered Tissues in Standard C (The University of Texas M. D. Anderson Cancer Center, Houston, Texes, 1992).

5. L.-H. Wang, and S. L. Jacques, "Animated simulation of light transport in tissues," Proc. Soc. Photo-Opt. Instrum. Eng. 2134A, 247-254 (1994). 